\title{
A Devastating Complication of Vivax Malaria: Acute Kidney Injury and Gangrene Feet
}

\author{
Rubina Naqvi, Hania Siddique, Syeda Shireen Taqvi \\ Department of Nephrology \\ Sindh Institute of Urology and Transplantation (SIUT), \\ Karachi, Pakistan

\begin{abstract}
Abstarct: usual antimalarials and complications are rare, yet occasionally severe complications are observed including Acute kidney injury.
\end{abstract} \\ Malarial infestation is common in Pakistan and Plasmodium vivax is a common causative organism. Although it has been managed successfully with
}

We present her a case of AKI secondary to plasmodium vivax along with gangrene of bilateral feet.

Keywords: Vivax, plasmodium, complications, AKI, acute kidney injury, gangrene, microangiopathy, ATN, pigment nephropathy.

\section{Corresponding Author}

Professor Dr. Rubina Naqvi

Department of Nephrology

SIUT,

Karachi Pakistan

Email: rubinanaqvi@gmail.com, naqvirubina@yahoo.com

Received: February 10,2020. Accepted March 9,2020.

PJKD 2020;4(2):254-256

\section{Introduction:}

Plasmodium Vivax (P. Vivax) is no more 'benign tertiary malaria' , as it historically used to be, multi organ involvement with severe infection is well recognized fact and is reported from many countries where malaria is endemic. ${ }^{1-5}$

The sequences of pathognomonic mechanisms are reported as parasitized red cells becoming sticky and tend to adhere to adjacent healthy erythrocytes as well as platelets and the capillary endothelium. As a result there is intravascular rosettes and clumps formation, which can block the microcirculation. These mechanisms of damage do not stop here and from endothelial activation resulting in release of various cytokines and local mediators that may enhance peripheral capillary damage.

We hereby report a case of $\mathrm{P}$. vivax malaria complicated with acute kidney injury (AKI) and severe microangiopathy causing gangrene of feet and tip of nose.

\section{Case Report:}

A 15 year female, resident of village near a town in interior of Sindh Province, Pakistan was in previous good health when she developed fever 2 weeks prior to coming to our hospital. Fever was high grade, was not associated with much shivering but she had vomiting 3-4 episodes/ day. Developed anuria and taken to bigger city for treatment where found to have renal dysfunction and underwent two sessions of hemodialysis before reaching to our hospital. She had developed black color discoloration of both feet and tip of nose 3 days earlier. Her physical examination on presentation revealed temperature of $100^{\circ} \mathrm{F}, \mathrm{BP} 100 / 70$, Pulse of 92 beats per minute, pallor, bilateral pedal edema, gangrene of nasal tip and bilateral feet (toes and dorsum), Fig. 1. Rest of general and systemic examination was unremarkable.

Her laboratory investigations at presentation revealed; Hb $6.7 \mathrm{mg} / \mathrm{dL}$, TLC 31.4/ $\mathrm{mm}^{3}$ (Neutrophils: 58\%, Lymphocytes : $36 \%$ ), Platelet 75,000/mm $\mathrm{mm}^{3}$, PT 11/10.5, APTT 24.2/25.8, INR 1.04, MP positive for P.vivax, both Trophozoites and Gametocytes seen on peripheral film (percentage of parasitemia is not reported with vivax malaria, thus not available here), urea $153 \mathrm{mg} / \mathrm{dL}, \mathrm{creatinine} 6.96 \mathrm{mg} / \mathrm{dL}, \mathrm{Na}$ $134 \mathrm{mEq} / \mathrm{L}, \mathrm{K} 4.5 \mathrm{mEq} / \mathrm{L}, \mathrm{Cl} 96 \mathrm{mEq} / \mathrm{L}, \mathrm{HCO} 318 \mathrm{mEq} / \mathrm{L}, \mathrm{LDH} 1536 \mathrm{U} / \mathrm{L}, \mathrm{Ca} 7.69 \mathrm{mg} / \mathrm{dL}$, Phosphate $5.23 \mathrm{mg} / \mathrm{dL}$, uric acid 13.5 $\mathrm{mg} / \mathrm{dL}$, Bilirubin $1.56 \mathrm{mg} / \mathrm{dL}$, Direct 0.92, SGOT $56 \mathrm{U} / \mathrm{L}$, SGPT $81 \mathrm{U} / \mathrm{L}$, gamma GT 91U/L, Alkaline Phosphatase 306 U/L, Albumin $2.2 \mathrm{mg} / \mathrm{dL}$, Globulin 3.6, CPK 569 U/L. Urine analysis: Prot 2+ and numerous RBCs, urine C/S revealed no growth. Direct Coombs test was negative, C3 0.96 (0.79-1.52 g/L), C4 0.15 (0.16-0.38 g/L), ANA negative, ASOT 63, RA factor negative, Anti Phospholipid Antibody (APLA) $\beta 2$ GP1 IgG positive 23.25 units ( $<20$ negative), Anti cardiolipin IgG positive with $35.66 \mathrm{U} / \mathrm{ml}$, Anti cardiolipin IgM positive 
with $29.17 \mathrm{U} / \mathrm{ml}$ (<20 indeterminate), rest negative. HBV and HCV serology was negative. Renal Biopsy was performed after one week of hospitalization and it revealed acute tubular necrosis with pigment casts in some tubular lumina, Fig. 2.

Figure 1: Photographed patients nasal and feet lesions (with permission)

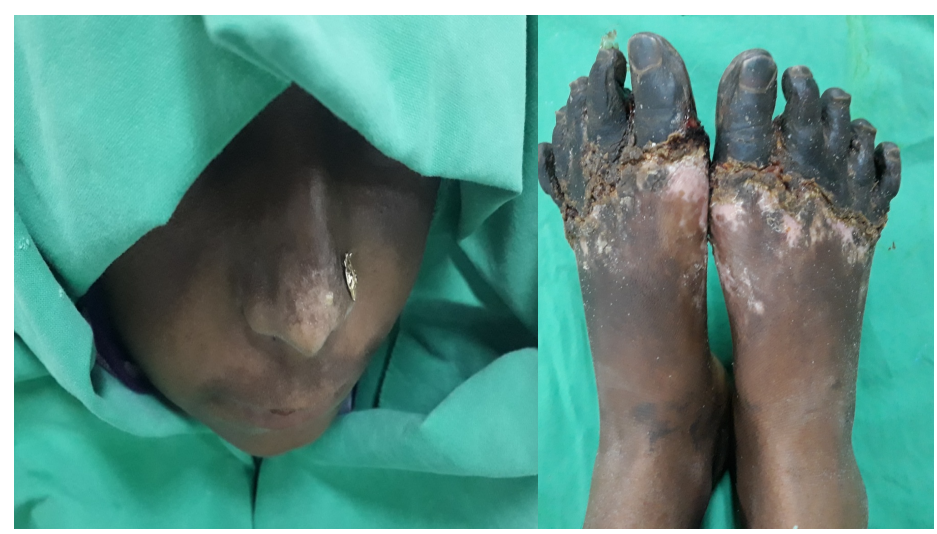

Figure 2: Photomicrograph showing a normal glomerulus. The surrounding tubules show Acute Tubular Necrosis (ATN) and pigment casts in some tubular lumena. (H\&E, $\times 200)$.

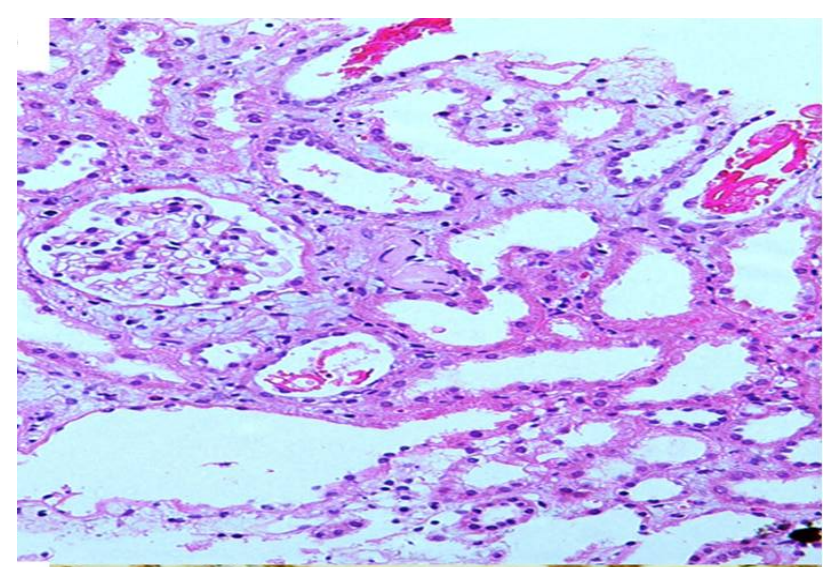

Color Doppler Imaging on day of admission showed no evidence of arterial insufficiency in both legs; femoral, popliteal, anterior and post tibial arteries were traced during exam. Dorsalis pedis on right was of normal velocity, on left could not assessed because of edema. Chest X-Ray and Ultra Sonogram of whole abdomen were normal. (Spleen $11.2 \mathrm{~cm}$ ) ECG was normal.

Patient required seven sessions of hemodialysis before her urine output markedly improved. She had a prolonged stay because of infected wound of feet and required bilateral amputation of fore feet.

She did not require hemodialysis at discharge and her serum creatinine was $3.2 \mathrm{mg} / \mathrm{dl}$ (eGFR of $21.7 \mathrm{ml} / \mathrm{min}$ ) on last follow up at 17 weeks post discharge. Being CKD 4 arterio- venous fistula was created.

\section{Discussion:}

According to WHO published fact sheet for malaria; in 2017 there were an estimated 219 million cases from 87 countries. The estimated number of malaria deaths were 435,000 in the year 2017. 'This included infections from all species of plasmodium. While Plasmodium falciparum is responsible for more deaths, Plasmodium vivax is the most widespread of all of the malarial species, and can also cause severe and fatal infections adding significantly to global morbidity and mortality.

P. vivax has been reported to cause severe anemia, respiratory distress, malnutrition, neurological symptoms possibly coma. Recurrent hemolysis and dyserythropoesis from relapsing vivax malaria may intensify anemia. There have been various case reports of noncardiogenic pulmonary edema/acute lung injury in patients with vivax malaria. ${ }^{9}$ Another rare complication of vivax malaria is spleenic rupture which carries a mortality rate of up to $80 \% .^{10}$

AKI from P.Vivax infection has been previously reported from neighboring country India as well as from our own institution and other renal care tertiary unit from our own country and is now established reported complication from this species of malaria. 3,4,10,11 
A study from country; based on 107 patients with vivax malaria and addressing significant morbidity with this species of malaria has reported thrombocytopenia in $61.7 \%$, anemia in $21.5 \%$ and renal dysfunction in $3.73 \%$ cases. $^{12}$

Another published study from country, reported thrombocytopenia in $91.9 \%$ of 296 patients with vivax malaria and multi organ dysfunction (MOD) in 7.1\% cases which is slightly lower from plasmodium falciparum which showed MOD in 10.6\% cases in same study. ${ }^{13}$

Also found in literature is hemolytic uremic syndrome (HUS), severe microangiopathy and autoimmune hemolytic anemia as case reports. ${ }^{14,15}$ Among many causes of anemia; malaria has been reported as a major cause in tropical countries. It can cause hemolysis of both infected and uninfected red blood cells and bone marrow dyserythropoiesis which compromises rapid recovery from anemia. ${ }^{16} \mathrm{~A}$ case report of 11 year boy with vivax malaria complicated by anemia, thrombocytopenia, anuric renal failure secondary to thrombotic microangiopathy, and acute respiratory distress syndrome has been reported from India. ${ }^{17}$

A collaborative study done at malaria research laboratories in Brazil and US, has highlighted correlation of malaria immunity with autoimmunity and phospholipid antibody reacting antibodies. The authors of this particular study could not find a relationship between the presence of anti-glycosylphosphatidylinositol (GPI) antibodies in the serum and asymptomatic malaria infection, but they reported positive relationship between these antibodies and infected malaria patients. ${ }^{18}$ Our patient had shown positive APLA $\beta 2$ GP1 IgG, Anti cardiolipin IgG and Anti cardiolipin IgM.

Our patient had severe microangiopathy, parasitized red blood cells plugging distal most capillary bed causing gangrene in fore feet and tip of nose, ischemic tubular necrosis was most probable cause of AKI in this patient (which is also evident from histopathology report), along with other hypothetical mechanisms might be contributing at same time. Renal recovery with acute tubular necrosis and pigment nephropathy is generally good but unfortunately this patient developed infected wound in gangrenous feet and with prolonged hospitalization acquired multi drug resistance in isolated bacteria from pus oozing from wound, thus requiring antibiotics which are more nephrotoxic. Though became dialysis free she does not achieve complete renal recovery and would remain in need of lifelong CKD therapy.

\section{References:}

1. Kochar DK, Das A, Kochar SK, Saxena V, Sirohi P, Garg S,et al. Severe Plasmodium vivax Malaria: A Report on Serial Cases from Bikaner in Northwestern India. Am J Trop Med Hyg. 2009;80(2):194 - 198.

2. Mishra SK, Das BS. Malaria and Acute Kidney Injury. Semin in Nephrology, 2008; 28(4):395-408

3. Naqvi R, Akhtar F, Ahmed E, Sheikh R, Bhatti S, Haider A, Naqvi A, Rizvi A. Malarial Acute Kidney Injury: 25 Years Experience from a Center in an Endemic Region. British Journal of Medicine \& Medical Research. 2016;12(6): 1-6, Article no.BJMMR.21471

4. $\quad$ Naqvi R.Plasmodium Vivax causing acute kidney injury: A foe less addressed. Pak J Med Sci 2015; 31(6):1472-5

5. Price RN, Tjitra E, Guerra CA, Yeung S, Nicholas J White NJ,Anstey NM. Vivax malaria: neglected and not benign. Am J Trop Med Hyg. 2007 ; 77 (6 Suppl): $79-87$

6. Barsoum RS. Malarial Acute Renal Failure. J Am Soc Nephrol. 2000;11:2147 - 2154

7. www.WHO World Malaria report 2018. Accessed on $27^{\text {th }}$ Jan.2020

8. Lacey Menkin-Smith L, Winders WT. Malaria (Plasmodium Vivax). Last Update: May 4, 2019. Accessed on $27^{\text {th }}$ Jan. 2020 https://www.ncbi.nlm.nih.gov/books/NBK538333/

9. Hartley J. Oxford handbook of tropical medicine. BMJ. 2000;320(7236):721A

10. Prakash J, Singh AK, Kumar NS, Saxena RK. Acute Renal Failure in Plasmodium vivax Malaria. JAPI. 2003;51:265-267.

11. Imtiaz S, Drohlia MF, Nasir K, Hussain M, Ahmad A. Morbidity and Mortality Associated with Plasmodium Vivax and Plasmodium Falciparum Infection in a Tertiary Care Kidney Hospital. Saudi J Kid DisTrans. 2015; 26(6):1169-76

12. Aatif S, Jamal Q, Altaf A, Salimullah. Is vivax malaria really benign? - A Karachi-based study. J Pak Med Assoc. 2013;63(6):721-4.

13. Zubairi ABS, Nizami S, Raza A, Mehraj V, Rasheed AF, Ghanchi NK, Khaled ZN, Beg MA. Severe Plasmodium vivax Malaria in Pakistan. Emerg Infectious Dis. 2013;19(11):1851-4.

14. Keskar VS, Jamale TE, Hase NK. Hemolytic uremic syndrome associated with Plasmodium vivax malaria successfully treated with plasma exchange. Indian J Nephrol. 2014;24(1): 35 - 37.

15. Singh D, Gupta V, Acharya S, Mahajan SN, Verma A. A case of Plasmodium vivax malaria associated with severe autoimmune hemolytic anaemia. Ann Trop Med Public Health 2012;5:133-6

16. White NJ. Anaemia and Malaria. Malar J. 2018;17(1):371.

17. Saharan S, Kohli U, Lodha R,Sharma A, Bagga A. Thrombotic microangiopathy associated with Plasmodium vivax malaria. Pediatr Nephrol. 2009; 24:623 - 4.

18. Gomes LR, Martins YC, Ferreira-da-Cruz MF, Daniel-Ribeiro CT. Autoimmunity, phospholipid-reacting antibodies and malaria immunity. Lupus. 2014;23(12):1295-8. 